Article

\title{
Dual Superconductor Model of Confinement: Quantum-String Representation of the 4D Yang-Mills Theory on a Torus and the Correlation Length away from the London Limit
}

\author{
Dmitry Antonov
}

check for updates

Citation: Antonov, D. Dual

Superconductor Model of

Confinement: Quantum-String

Representation of the 4D Yang-Mills

Theory on a Torus and the

Correlation Length away from the

London Limit. Universe 2022, 8, 7 .

https://doi.org/10.3390/

universe 8010007

Academic Editor: Ralf Hofmann

Received: 15 November 2021

Accepted: 21 December 2021

Published: 24 December 2021

Publisher's Note: MDPI stays neutral with regard to jurisdictional claims in published maps and institutional affiliations.

Copyright: (C) 2021 by the author. Licensee MDPI, Basel, Switzerland. This article is an open access article distributed under the terms and conditions of the Creative Commons Attribution (CC BY) license (https:/ / creativecommons.org/licenses/by/ $4.0 /)$.
Formerly at Departamento de Física and CFIF, Instituto Superior Técnico, ULisboa, Av. Rovisco Pais, 1049-001 Lisbon, Portugal; dr.dmitry.antonov@gmail.com

\begin{abstract}
This paper is devoted to the dual superconductor model of confinement in the 4D Yang-Mills theory. In the first part, we consider the latter theory compactified on a torus, and use the dual superconductor model in order to obtain the Polchinski-Strominger term in the string representation of a Wilson loop. For a certain realistic critical value of the product of circumferences of the compactification circles, which is expressed in terms of the gluon condensate and the vacuum correlation length, the coupling of the Polchinski-Strominger term turns out to be such that the string conformal anomaly cancels out, making the string representation fully quantum. In the second part, we use the analogy between the London limit of the dual superconductor and the low-energy limit of the $4 \mathrm{D}$ compact QED, to obtain the partition function of the dual superconductor model away from the London limit. There, we find a decrease of the vacuum correlation length, and derive the corresponding potential of monopole currents.
\end{abstract}

Keywords: effective-string description of confinement; models of the non-perturbative Yang-Mills vacuum; color confinement; Yang-Mills theory; finite-temperature effects in quantum field theory; magnetic monopoles; Wilson loop

\section{Introduction}

Dual superconductor is known to be one of the earliest and well established models of quark confinement in QCD [1,2] (for a review, see [3]). It suggests a model for the Yang-Mills vacuum which is based on the condensate of a magnetically charged Higgs field. The corresponding dual Abelian Higgs model (DAHM) represents a $4 \mathrm{D}$ relativistic generalization of the Landau-Ginzburg theory of dual superconductivity. This scenario of confinement is fully supported by the dedicated lattice simulations [4,5]. One has to realize, though, that the monopole condensation provides confinement of only the diagonal degrees of freedom, through the violation of the Abelian Bianchi identities. In this sense, the dual-superconductor model differs from the Yang-Mills theory, where confinement (of both diagonal and off-diagonal degrees of freedom) does not require violation of either Abelian or non-Abelian Bianchi identities. That is, in the dual superconductor, confinement is described in terms of a field dual to that of the dual vector boson, i.e., an antisymmetrictensor field $h_{\mu \nu}$ which couples to a world sheet bounded by the contour of the 't Hooft loop (for a review, see [6]). Unlike the gauge-field strength tensor, the field $h_{\mu \nu}$ contains an additional term $\propto \varepsilon_{\mu \nu \lambda \rho}$, i.e., $h_{\mu \nu}=\partial_{\mu} B_{v}-\partial_{\nu} B_{\mu}+\varepsilon_{\mu \nu \lambda \rho} \partial_{\lambda} \varphi_{\rho}$, which violates the Abelian Bianchi identities. This term yields the tensor structure $\left(\delta_{\mu \lambda} \delta_{\nu \rho}-\delta_{\mu \rho} \delta_{\nu \lambda}\right)$ in the correlation function $\left\langle h_{\mu \nu}(x) h_{\lambda \rho}(y)\right\rangle$, which, in turn, yields confinement. (The same is true for compact QED [7].) Rather, the non-Abelian field-strength tensor $F_{\mu \nu}^{a}$ does not contain a term $\varepsilon_{\mu \nu \lambda \rho} \partial_{\lambda} \varphi_{\rho}^{a}$, and yet the correlation function $\left\langle F_{\mu \nu}^{a}(x) F_{\lambda \rho}^{b}(y)\right\rangle$ contains the tensor structure $\left(\delta_{\mu \lambda} \delta_{\nu \rho}-\delta_{\mu \rho} \delta_{v \lambda}\right)$ [8,9] (for a review, see [10]). Consequently, in the non-Abelian case, one can still fix a certain gauge, e.g., the Fock-Schwinger gauge which expresses $A_{\mu}^{a}$ in terms of $F_{\mu \nu}^{a}$ (to obtain the propagator $\left\langle A_{\mu}^{a}(x) A_{\nu}^{b}(y)\right\rangle$ with the confinement effects, which can further 
be used in the non-local Nambu-Jona-Lasinio model [11] corresponding to QCD with light quarks in the Gaussian approximation), whereas this is no longer possible in a confining Abelian theory like the dual superconductor or compact QED.

The fundamentally important problem for the description of confinement is the construction of a string represenation of the Wilson loop in the Yang-Mills theory, or of the 't Hooft loop in DAHM (for a review, see e.g., [6]). The partition function of DAHM, in the London limit, admits such a representation in terms of closed dual Abrikosov-NielsenOlesen strings. Indeed, as the dual Higgs field has singularities at the cores of those strings, one can unambiguously reformulate the integration over the phase of the Higgs field in terms of the integration over the strings' world sheets. Moreover, it turns out that the Jacobian, corresponding to this change of variables in the functional integral, yields the Polchinski-Strominger term [12] with such a coefficient that the string conformal anomaly (for a review, see [13]) gets cancelled out in 4D rather than 26D [14]. While this is certainly a successful approach to the construction of a fully quantum string representation of the DAHM partition function, it remains unclear whether, in the Yang-Mills theory, a representation of the Wilson loop in terms of an integral over open-string world sheets can be constructed in a similar way. The main obstacle in this way is the identification, in the Yang-Mills integration measure, of those degrees of freedom that can be related to the string's world-sheet coordinates. In a recent paper [15], an alternative approach has been proposed, where the Polchinski-Strominger term emerges from the quartic cumulant in the cumulant expansion of the Wilson loop. That has been done in the 3D Yang-Mills theory at finite temperature, with the correlations between the elements of the string's world sheet modeled by those of the London limit of DAHM, which can be calculated analytically. The corresponding finite-temperature compactification of the theory is important for the generation of the Polchinski-Strominger term and the cancellation of the string conformal anomaly at a certain temperature, which turns out to be approximately twice smaller than the deconfinement critical temperature. In the first part of the present paper, this approach will be generalized to the case of the $4 \mathrm{D}$ Yang-Mills theory compactified on a torus, so that it can, in particular, become applicable to the zero-temperature theory in a space with two compact spatial dimensions.

In the second part of the paper, another DAHM-related issue will be addressed, which is the calculation of the correlation length in that theory away from the London limit. In this way, an analogy between the dual representations of the London limit of DAHM and the low-energy limit of $4 \mathrm{D}$ compact QED turns out to be helpful, because of the known Higgs-induced corrections to the 3D compact QED, which take place when the latter theory emerges from the 3D Georgi-Glashow model. In the Summary section, some concluding remarks will be presented.

\section{Quantum-String Representation of the Wilson Loop in the Yang-Mills Theory Compactified on a Torus}

Let us consider the Yang-Mills theory compactified on a torus, which is, e.g., the case if this theory is compactified on $\mathbb{R}^{3} \times S^{1}$ [16] and further taken at finite temperature [17], or just in the continuum limit of a lattice version of the theory with periodic boundary conditions (for a review, see e.g., [18]). In such a compactified version of the Yang-Mills theory, we are interested in the string representation of a Wilson loop corresponding to a static quark and an antiquark separated along one of the non-compact dimensions (as opposed to the so-called torelon, which is a closed string emerging in case when that dimension is compact [19]). Let us further model the confining part of the two-point correlation function of gluonic field strengths by the corresponding expression for the dual field strengths in the London limit of DAHM, which reads [20,21] (for a review, see [22]) $\left\langle F_{\mu \nu}(x) F_{\lambda \rho}(0)\right\rangle_{\text {conf }}=\left(\delta_{\mu \lambda} \delta_{v \rho}-\delta_{\mu \rho} \delta_{v \lambda}\right) \mathcal{D}(x)$, where $\mathcal{D}(x)=\frac{m^{4}}{4 \pi^{2}} \frac{K_{1}(m|x|)}{m|x|}$. Here, $m=g_{m} \eta$ is 
the mass of the dual vector boson, with $g_{m}$ being the magnetic coupling constant, and $\eta$ being the v.e.v. of the dual Higgs field. Note that $\mathcal{D}(x)=m^{2} D_{m}(x)$, where:

$$
D_{m}(x)=\frac{m}{4 \pi^{2}} \frac{K_{1}(m|x|)}{|x|}
$$

is just the massive-boson propagator, which obeys the equation $\left(-\partial^{2}+m^{2}\right) D_{m}(x)=\delta(x)$, and $K_{v}(x)$ henceforth denotes a Macdonald function, which is a modified Bessel function of the second kind. Due to the compactification, we further have $x^{2}=\mathbf{R}^{2}+(\alpha n)^{2}+\left(\beta n^{\prime}\right)^{2}$. Here, $\mathbf{R}$ is a $2 \mathrm{D}$ vector in the non-compact dimensions, $\alpha$ and $\beta$ are the circumferences of the two circles which are forming the torus, and $n$ and $n^{\prime}$ are the winding numbers. We further use integral representation $\frac{K_{1}(m|x|)}{m|x|}=\int_{0}^{\infty} \mathrm{d} t \mathrm{e}^{-\frac{1}{4 t}-t(m x)^{2}}$, along with the following generalization of the Poisson sum formula, which can be considered as a discrete version of the Gaussian integral [23]:

$$
\sum_{n=-\infty}^{\infty} \mathrm{e}^{-t(m \alpha n)^{2}}=\frac{\sqrt{\pi / t}}{m \alpha} \sum_{k=-\infty}^{\infty} \mathrm{e}^{-\left(\frac{\pi k}{m \alpha}\right)^{2} / t}
$$

The subsequent $t$-integration yields

$$
\int_{0}^{\infty} \frac{\mathrm{d} t}{\sqrt{t}} \mathrm{e}^{-t\left(m L_{n^{\prime}}\right)^{2}-\frac{1}{4 t}\left(1+\left(\frac{2 \pi k}{m \alpha}\right)^{2}\right)}=\frac{\sqrt{\pi}}{m L_{n^{\prime}}} \mathrm{e}^{-M_{k} L_{n^{\prime}}},
$$

where $L_{n^{\prime}} \equiv \sqrt{\mathbf{R}^{2}+\left(\beta n^{\prime}\right)^{2}}$ and $M_{k} \equiv m \sqrt{1+\left(\frac{2 \pi k}{m \alpha}\right)^{2}}$. Applying now the same generalized Poisson sum formula once again, in the form

$$
\sum_{n^{\prime}=-\infty}^{\infty} \frac{\mathrm{e}^{-M_{k} L_{n^{\prime}}}}{L_{n^{\prime}}}=\frac{2}{\beta} \sum_{k^{\prime}=-\infty}^{\infty} K_{0}\left(R \sqrt{\left(M_{k}\right)^{2}+\left(2 \pi k^{\prime} / \beta\right)^{2}}\right),
$$

we obtain the following double-sum representation for the function $\mathcal{D}(x)$ :

$$
\mathcal{D}(x)=\frac{m^{2}}{2 \pi \alpha \beta} \sum_{k, k^{\prime}=-\infty}^{\infty} K_{0}\left(m R \sqrt{1+\left(\frac{2 \pi k}{m \alpha}\right)^{2}+\left(\frac{2 \pi k^{\prime}}{m \beta}\right)^{2}}\right) .
$$

As long as $\left(\frac{2 \pi}{m \alpha}\right)^{2}+\left(\frac{2 \pi}{m \beta}\right)^{2} \gtrsim 1$, this expression can be approximated as:

$$
\mathcal{D}(x) \simeq \frac{m^{2}}{2 \pi \alpha \beta} K_{0}(m R),
$$

since all the terms with a non-zero $k$ or $k^{\prime}$ become exponentially suppressed.

By equating to each other the string tensions in the DAHM in the London limit and in the Yang-Mills theory in the Gaussian approximation, we will now establish a correspondence between these two theories. The string tension in the DAHM in the London limit, corresponding to the string interconnecting two unit electric charges, stems from the non-local string action

$$
S=\frac{1}{8} \int \mathrm{d} \sigma_{\mu \nu}(x) \int \mathrm{d} \sigma_{\lambda \rho}\left(x^{\prime}\right)\left\langle F_{\mu \nu}(x) F_{\lambda \rho}\left(x^{\prime}\right)\right\rangle_{\text {conf }}=\frac{1}{4} \int \mathrm{d} \sigma_{\mu \nu}(x) \int \mathrm{d} \sigma_{\mu \nu}\left(x^{\prime}\right) \mathcal{D}\left(x-x^{\prime}\right)
$$

and reads [20-22]

$$
\sigma=\frac{1}{2 m^{2}} \int \mathrm{d}^{2} z \mathcal{D}(\mathbf{z})=\frac{m^{2}}{8 \pi^{2}} \int \mathrm{d}^{2} z \frac{K_{1}(|\mathbf{z}|)}{|\mathbf{z}|} \simeq \frac{m^{2}}{4 \pi} \ln \frac{M}{m},
$$


with $M$ standing for the Higgs mass. The last equality here is valid within the leading logarithmic approximation to the London limit [24,25], which assumes that not only $\frac{M}{m} \gg 1$, but also $\ln \frac{M}{m} \gg 1$. On the other hand, in the Yang-Mills theory in the Gaussian approximation, the non-local string action, with the exponential parametrization for the function $\mathcal{D}(x)$, reads $[8-10]$

$$
S=\kappa \frac{\left\langle\left(g F_{\mu \nu}^{a}\right)^{2}\right\rangle}{96 N} \int \mathrm{d} \sigma_{\mu \nu}(x) \int \mathrm{d} \sigma_{\mu \nu}\left(x^{\prime}\right) \mathrm{e}^{-m\left|x-x^{\prime}\right|} .
$$

In this expression, $N$ is the number of colors, $g$ is the Yang-Mills coupling, and we have directly set the Yang-Mills vacuum correlation length equal to $\frac{1}{m}$. Furthermore, parameter $\kappa$, which determines the mixing between the confining- and the non-perturbative non-confining contributions to the correlation function $\left\langle F_{\mu \nu}^{a}(x) F_{\lambda \rho}^{b}(y)\right\rangle$, is numerically equal to $0.83 \pm 0.03$ in the $\mathrm{SU}(3)$-case of interest [26], so we set $\kappa \simeq 0.83$. The resulting string tension reads $\sigma=\frac{\pi \kappa\left\langle\left(g F_{\mu \nu}^{a}\right)^{2}\right\rangle}{24 m^{2}}$, yielding the following correspondence:

$$
\ln \frac{M}{m}=\frac{\pi^{2} \kappa\left\langle\left(g F_{\mu \nu}^{a}\right)^{2}\right\rangle}{6 N m^{4}}
$$

Let us now replace $\mathrm{e}^{-m|x|}$ by $A \frac{K_{1}(m|x|)}{m|x|}$ in the parametrization of the correlation function $\left\langle F_{\mu \nu}^{a}(x) F_{\lambda \rho}^{b}(y)\right\rangle$. That yields for the normalization coefficient $A$ :

$$
A \equiv A(m)=\frac{1}{\ln \frac{M}{m}}=\frac{6 N m^{4}}{\pi^{2} \kappa\left\langle\left(g F_{\mu \nu}^{a}\right)^{2}\right\rangle} .
$$

Following the approach of Ref. [15], we will further make the same replacement in the confining structure of the quartic cumulant [27],

$$
\begin{gathered}
\left\langle g^{4} F_{\mu_{1} v_{1}}^{a_{1}}\left(x_{1}\right) F_{\mu_{2} v_{2}}^{a_{2}}\left(x_{2}\right) F_{\mu_{3} v_{3}}^{a_{3}}\left(x_{3}\right) F_{\mu_{4} v_{4}}^{a_{4}}\left(x_{4}\right)\right\rangle_{c}=C\left\langle\left(g^{2} F_{\mu v}^{a} F_{\mu \nu}^{a}\right)^{2}\right\rangle \times \\
\times\left[\delta^{a_{1} a_{2}} \delta^{a_{3} a_{4}}\left(\delta_{\mu_{1} \mu_{2}} \delta_{v_{1} v_{2}}-\delta_{\mu_{1} v_{2}} \delta_{\mu_{2} v_{1}}\right)\left(\delta_{\mu_{3} \mu_{4}} \delta_{v_{3} v_{4}}-\delta_{\mu_{3} v_{4}} \delta_{\mu_{4} v_{3}}\right)+\right. \\
+\delta^{a_{1} a_{3}} \delta^{a_{2} a_{4}}\left(\delta_{\mu_{1} \mu_{3}} \delta_{v_{1} v_{3}}-\delta_{\mu_{1} v_{3}} \delta_{\mu_{3} v_{1}}\right)\left(\delta_{\mu_{2} \mu_{4}} \delta_{v_{2} v_{4}}-\delta_{\mu_{2} v_{4}} \delta_{\mu_{4} v_{2}}\right)+ \\
\left.+\delta^{a_{1} a_{4}} \delta^{a_{2} a_{3}}\left(\delta_{\mu_{1} \mu_{4}} \delta_{v_{1} v_{4}}-\delta_{\mu_{1} v_{4}} \delta_{\mu_{4} v_{1}}\right)\left(\delta_{\mu_{2} \mu_{3}} \delta_{v_{2} v_{3}}-\delta_{\mu_{2} v_{3}} \delta_{\mu_{3} v_{2}}\right)\right] \times \\
\times \mathrm{e}^{-m\left(\left|z_{12}\right|+\left|z_{13}\right|+\left|z_{14}\right|+\left|z_{23}\right|+\left|z_{24}\right|+\left|z_{34}\right|\right)},
\end{gathered}
$$

where $z_{i j}=x_{i}-x_{j}$ are the relative coordinates of the points $x_{1}, \ldots, x_{4}$, and

$$
C=\left\{\left(N^{2}-1\right)\left(D^{2}-D\right)\left[\left(N^{2}-1\right)\left(D^{2}-D\right)+4\right]\right\}^{-1} .
$$

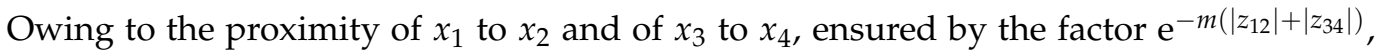
we have:

$$
\begin{gathered}
\mathrm{e}^{-m\left(\left|z_{12}\right|+\left|z_{13}\right|+\left|z_{14}\right|+\left|z_{23}\right|+\left|z_{24}\right|+\left|z_{34}\right|\right)} \simeq \\
\simeq \mathrm{e}^{-m\left(\left|z_{12}\right|+\left|z_{34}\right|+4\left|z_{13}\right|\right)} \simeq A^{2}(m) A(4 m) \frac{K_{1}\left(m\left|z_{12}\right|\right)}{m\left|z_{12}\right|} \frac{K_{1}\left(m\left|z_{34}\right|\right)}{m\left|z_{34}\right|} \frac{K_{1}\left(4 m\left|z_{13}\right|\right)}{4 m\left|z_{13}\right|} .
\end{gathered}
$$

By using Equations (2) and (4), we further have for this expression:

$$
\left(\frac{6 N m^{4}}{\pi^{2} \kappa\left\langle\left(g F_{\mu \nu}^{a}\right)^{2}\right\rangle}\right)^{3} \cdot 64 \cdot\left(\frac{2 \pi}{m^{2} \alpha \beta}\right)^{3} \cdot \frac{1}{16} K_{0}\left(m\left|\mathbf{z}_{12}\right|\right) K_{0}\left(m\left|\mathbf{z}_{34}\right|\right) K_{0}\left(4 m\left|\mathbf{z}_{13}\right|\right) .
$$


Setting now $N=3$ and $D=4$, we obtain the following Polchinski-Strominger term [12] in the string representation of a Wilson loop (cf. Reference [15]):

$$
S=-\frac{1}{m^{2}\left\langle\left(g F_{\mu \nu}^{a}\right)^{2}\right\rangle(\alpha \beta)^{3}} \frac{24}{25 \kappa^{3}} \int \mathrm{d}^{2} \xi \int \mathrm{d}^{2} \xi^{\prime} \sqrt{g} \mathcal{R}\left(-\frac{1}{\partial^{2}}\right)_{\xi, \xi^{\prime}} \sqrt{g^{\prime}} \mathcal{R}^{\prime} .
$$

Here, $\partial^{2} \equiv \partial^{a} \partial_{a}$ and $\mathcal{R}=-\mathrm{e}^{-\varphi} \partial^{2} \varphi$ are the expressions for the Laplacian $\Delta=\frac{1}{\sqrt{g}} \partial_{a} \sqrt{g} g^{a b} \partial_{b}$ and the scalar curvature of the world sheet in the conformal gauge, where the induced metric $g_{a b}=\partial_{a} x_{\mu} \cdot \partial_{b} x_{\mu}$ has the diagonal form $g_{a b}=\sqrt{g} \delta_{a b}$, and $\varphi=\ln \sqrt{g}$ with $g \equiv \operatorname{det} g_{a b}$. Furthermore, $\left(-\frac{1}{\partial^{2}}\right)_{\xi, \xi^{\prime}}=\frac{1}{2 \pi} \ln \frac{1}{\left|\xi-\xi^{\prime}\right|}$ is the Green's function of the Laplacian, and we have denoted $g^{\prime}=g\left(\boldsymbol{\xi}^{\prime}\right)$ and $\mathcal{R}^{\prime}=\mathcal{R}\left(\boldsymbol{\xi}^{\prime}\right)$. We have also used the facts that, for $c>0$, $K_{0}\left(c\left|\xi-\xi^{\prime}\right|\right) \rightarrow 2 \pi\left(-\frac{1}{\partial^{2}}\right)_{\xi, \xi^{\prime}}$ at $\xi \rightarrow \xi^{\prime}$, and that $\int \mathrm{d}^{2} z \mathbf{z}^{2} K_{0}(|\mathbf{z}|)=8 \pi$.

For the Polchinski-Strominger term (6) to cancel the bosonic-string conformal anomaly in $4 \mathrm{D}$, the coefficient in front of the integral in Equation (6) should be equal to $-\frac{26-4}{96 \pi}$ (cf. Refs. [6,15]). This yields the following relation for the circumferences of the compactification circles, whose fulfillment provides the cancellation of the conformal anomaly:

$$
\alpha \beta=\frac{1}{\kappa}\left(\frac{1152 \pi}{275 m^{2}\left\langle\left(g F_{\mu \nu}^{a}\right)^{2}\right\rangle}\right)^{1 / 3} \simeq \frac{2.85}{\left(m^{2}\left\langle\left(g F_{\mu \nu}^{a}\right)^{2}\right\rangle\right)^{1 / 3}} .
$$

The gluon condensate, $\left\langle\left(g F_{\mu \nu}^{a}\right)^{2}\right\rangle$, calculated from the aforementioned expression for the string tension, $\sigma=\frac{\pi \kappa\left\langle\left(g F_{\mu \nu}^{a}\right)^{2}\right\rangle}{24 N m^{2}}$, by means of the phenomenological SU(3) values $\sigma=(440 \mathrm{MeV})^{2}$ and [28] $\frac{1}{m}=0.22 \mathrm{fm}$, reads $\left\langle\left(g F_{\mu \nu}^{a}\right)^{2}\right\rangle=4.3 \mathrm{GeV}^{4}$. That yields the numerical relation $\sqrt{\alpha \beta}=0.27 \mathrm{fm}$. Accordingly, we have $\left(\frac{2 \pi}{m \alpha}\right)^{2}+\left(\frac{2 \pi}{m \beta}\right)^{2} \geq\left(\frac{2 \pi}{m}\right)^{2} \cdot \frac{2}{\alpha \beta} \simeq 52.1 \gg 1$, which justifies approximation (2).

Note also that there is no contradiction with the fact that the compactification radii, $\frac{\alpha}{2 \pi}$ and $\frac{\beta}{2 \pi}$, can be smaller than the vacuum correlation length $\frac{1}{m}$, since the Wilson loop is extending in the non-compact dimensions. As for the string, its existence requires to have the size of at least one of the two compact dimensions larger than the vacuum correlation length. For example, in the case when one of the two compact dimensions is $x_{4}$, which corresponds to finite temperature $T=1 / \beta$, the spatial dimension with compactification radius $\frac{\alpha}{2 \pi}$ shrinks in the zero-temperature limit. Nevertheless, the string is still well defined in the resulting (2+1)D Yang-Mills theory. Note also that the limit of the small radius of spatial compactification is precisely the one where the Yang-Mills theory compactified on $\mathbb{R}^{3} \times S^{1}$ admits an analytic description of confinement [16]. In the opposite limit of large temperatures, $T \gtrsim 2 T_{c}$, with $T_{c}$ standing for the deconfinement critical temperature, where the Yang-Mills theory effectively becomes purely spatial, the obtained condition (7) can also be fulfilled, making the string representation of the corresponding spatial Wilson loop fully quantum. Indeed, in that limit, the chromomagnetic condensate is proportional to the fourth power of the inverse chromomagnetic-vacuum correlation length, $m \propto g^{2} T$, i.e., $[29,30]\left\langle\left(g F_{i j}^{a}\right)^{2}\right\rangle \propto\left(g^{2} T\right)^{4}$, where $g$ now stands for the finite-temperature Yang-Mills coupling, and $i$ and $j$ are the purely spatial indices. Therefore, in this high-temperature limit, condition (7) parametrically becomes $\alpha \beta \propto \frac{1}{\left(g^{2} T\right)^{2}}$. As follows from this relation, both $\beta=\frac{1}{T}$ and $\alpha \propto \frac{1}{g^{4} T}$ vanish at $T \gg T_{c}$, but $\alpha$ stays parametrically larger than the vacuum correlation length $\frac{1}{m}$, since $g \ll 1$. We notice that the thus emerged ultrasoft scale $\frac{1}{g^{4} T}$ defines the so-called sound attenuation length in the gluon plasma (see e.g., [31-33]) In particular, it can be shown $[34,35]$ that the correlation function $\left\langle F_{i j}^{a}(\vec{x}) F_{k l}^{b}(\overrightarrow{0})\right\rangle$ with the correlation length defined by that scale, yields the known perturbative expression for the shear viscosity of the plasma [36], $\eta \propto \frac{T^{3}}{g^{4} \ln \frac{\text { const }}{g}}$. The unexpected emergence of this ultrasoft scale, within the present context of the string representation of a spatial Wilson loop in the 
high-temperature limit, can be viewed as a remarkable example of possible interrelations between a priori different areas of physics of the Yang-Mills fields.

\section{DAHM away from the London Limit: Vacuum Correlation Length and the Distribution of Monopole Currents}

The partition function of $4 \mathrm{D}$ compact QED in the continuum limit has the form [22]:

$$
\mathcal{Z}_{4 \mathrm{D} \text { comp. QED }}=\int \mathcal{D} B_{\mu} \mathrm{e}^{-\int_{x}\left[\frac{1}{4} F_{\mu \nu}^{2}-2 \zeta \cos \left(\frac{\left|B_{\mu}\right|}{\Lambda}\right)\right]}
$$

where $B_{\mu}$ is the dual gauge field, $F_{\mu \nu}=\partial_{\mu} B_{\nu}-\partial_{\nu} B_{\mu}$ is its field-strength tensor, $\left|B_{\mu}\right| \equiv \sqrt{B_{\mu}^{2}}$, $\Lambda$ is the UV cutoff, $\zeta$ is the monopole fugacity of dimensionality (mass) ${ }^{4}$, the factor of 2 in front of it is due to the contributions of both monopoles and antimonopoles, and $\int_{x} \equiv \int \mathrm{d}^{4} x$. The Debye mass of the field $B_{\mu}$, stemming from this expression, is $m_{D}=\sqrt{2 \zeta} / \Lambda$. In the low-energy limit, which corresponds to the limit of low monopole densities, dualization of Equation (8) yields the following representation of the theory in terms of an antisymmetrictensor field $h_{\mu \nu}$ [22]:

$$
\mathcal{Z}_{\text {low }- \text { en. } 4 \text { D comp. QED }}=\int \mathcal{D} h_{\mu \nu} \mathrm{e}^{-\int_{x}\left(\frac{1}{12 m_{D}^{2}} H_{\mu \nu \lambda}^{2}+\frac{1}{4} h_{\mu \nu}^{2}\right)},
$$

where $H_{\mu v \lambda}=\partial_{\mu} h_{\nu \lambda}+\partial_{\lambda} h_{\mu v}+\partial_{v} h_{\lambda \mu}$ is the strength tensor of the field $h_{\mu v}$.

At the same time, the partition function of the London limit of DAHM (with closed dual Abrikosov-Nielsen-Olesen strings disregarded for this comparison, due to the absence of closed strings in compact QED) has the form [22]:

$$
\mathcal{Z}_{\mathrm{LL} \mathrm{of} \mathrm{DAHM}}=\int \mathcal{D} h_{\mu \nu} \mathrm{e}^{-\int_{x}\left(\frac{1}{12 m^{2}} H_{\mu \nu \lambda}^{2}+\frac{1}{4} h_{\mu \nu}^{2}\right)} .
$$

Thus, the two theories are equivalent to each other, with the equality $m_{D}=m$ yielding an expression for the fugacity,

$$
\zeta=\frac{(m M)^{2}}{2}
$$

where we have naturally set $\Lambda=M$.

Now, by an analogy with the 3D compact QED, we will proceed beyond the London limit of the $4 \mathrm{D}$ DAHM. In the case when the 3D compact QED is stemming from the 3D GeorgiGlashow model, for a not-infinitely-heavy Higgs field in the latter model (as compared to the mass of the W-boson), monopoles start interacting with each other via the Higgs field, so that their Coulomb interaction acquires a correction [37], $q_{a} q_{b} D_{0}\left(\vec{z}-\vec{z}^{\prime}\right)-D_{M}\left(\vec{z}-\vec{z}^{\prime}\right)$. Here, $q_{a}$ and $q_{b}$ are the charges (in the units of $g_{m}$ ) of two monopoles located at the points $\vec{z}$ and $\vec{z}^{\prime}, D_{0}(\vec{z})=1 /(4 \pi|\vec{z}|)$ is the dual-photon propagator, and $D_{M}(\vec{z})=\mathrm{e}^{-M|\vec{z}|} /(4 \pi|\vec{z}|)$ is the Higgs-boson propagator. We therefore expect that, in the 4D DAHM away from the London limit, the interaction between two monopoles, propagating along the world lines $z_{\mu}(\tau)$ and $z_{\mu}^{\prime}(\tau)$, becomes $\int \mathrm{d} \tau \mathrm{d} \tau^{\prime}\left[q_{a} q_{b} \dot{z}_{\mu} \dot{z}_{\mu}^{\prime} D_{0}\left(z-z^{\prime}\right)-\left|\dot{z}_{\mu}\right|\left|\dot{z}_{\mu}^{\prime}\right| D_{M}\left(z-z^{\prime}\right)\right]$, where $D_{0}(z)=1 /\left(4 \pi^{2} z^{2}\right), D_{M}(z)$ is given by Equation (1), and we have taken into account that the (dual) Higgs is a scalar particle. Hence, we can write down the following partition function of the DAHM away from the London limit (cf. the corresponding partition function stemming from the 3D Georgi-Glashow model with a not-infinitely-heavy Higgs, considered in Ref. [38]):

$$
\mathcal{Z}_{\text {DAHM beyond LL }}=\int \mathcal{D} B_{\mu} \mathcal{D} \varphi \mathrm{e}^{-\int_{x}\left[\frac{1}{4} F_{\mu v}^{2}+\frac{1}{2}\left(\partial_{\mu} \varphi\right)^{2}+\frac{M^{2}}{2} \varphi^{2}-(m M)^{2} \mathrm{e}^{\frac{\varphi}{M}} \cos \left(\frac{\left|B_{\mu}\right|}{M}\right)\right]}
$$

where we have once again set $\Lambda=M$, in $\mathrm{e}^{\frac{\varphi}{M}}$. The latter choice is natural, since the typical length of a monopole trajectory, $\int \mathrm{d} \tau\left|\dot{z}_{\mu}\right|$, is $\frac{1}{M}$. 
We further perform the $\varphi$-integration in Equation (9), by using the cumulant expansion in the Gaussian approximation (cf. Refs. [39,40]; for a review, see [41]), i.e.,

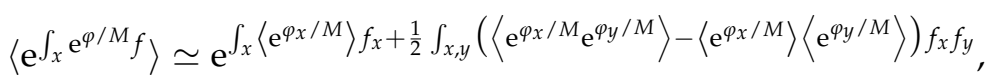

where $\langle\cdots\rangle \equiv \int \mathcal{D} \varphi \mathrm{e}^{-\frac{1}{2} \int_{x}\left[\left(\partial_{\mu} \varphi\right)^{2}+M^{2} \varphi^{2}\right]}(\cdots)$ and $f_{x} \equiv f(x)$. Averages entering this expression read $\left\langle\mathrm{e}^{\varphi_{x} / M}\right\rangle=\mathrm{e}^{D_{M}(0) /\left(2 M^{2}\right)}$ and $\left\langle\mathrm{e}^{\varphi_{x} / M} \mathrm{e}^{\varphi_{y} / M}\right\rangle=\mathrm{e}^{\left[D_{M}(0)+D_{M}(x-y)\right] / M^{2}}$. As we are always considering constant $m$, i.e., a constant Higgs v.e.v. $\eta$, by staying outside the cores of the dual Abrikosov-Nielsen-Olesen strings, $|x|>\frac{1}{M}$, which corresponds to having $\Lambda=M$, we should replace 0 by $1 / M$ in $D_{M}(0)$. This replacement yields $\mathrm{e}^{D_{M}(0) /\left(2 M^{2}\right)} \simeq \mathrm{e}^{K_{1}(1) /\left(8 \pi^{2}\right)}$. For the same reason of considering only the distances $|x|>\frac{1}{M}$, we can, with the exponential accuracy, approximate $\mathrm{e}^{D_{M}(x-y) / M^{2}} \simeq 1+\frac{1}{M^{2}} D_{M}(x-y)$. This yields for Equation (9):

$$
\mathcal{Z}_{\text {DAHM beyond LL }} \simeq \int \mathcal{D} B_{\mu} \mathrm{e}^{-\int_{x}\left[\frac{1}{4} F_{\mu v}^{2}-\mu^{4} \cos \left(\frac{|B \mu|}{M}\right)-\frac{\mu^{8}}{2 M^{2}} \int_{y} \cos \left(\frac{|B \mu(x)|}{M}\right) \cos \left(\frac{|B \mu(y)|}{M}\right) D_{M}(x-y)\right]},
$$

where $\mu^{4} \equiv(m M)^{2} \mathrm{e}^{K_{1}(1) /\left(8 \pi^{2}\right)} \simeq(m M)^{2}$, since $\mathrm{e}^{K_{1}(1) /\left(8 \pi^{2}\right)} \simeq 1.008$. Expanding now the product of the two cosines up to the quadratic term, $\int_{x, y}\left(1-\frac{B_{\mu}^{2}(x)}{2 M^{2}}\right)\left(1-\frac{B_{\mu}^{2}(y)}{2 M^{2}}\right) D_{M}(x-y) \simeq$ $-\frac{1}{M^{4}} \int_{x} B_{\mu}^{2}$, we obtain the following corrected squared Debye mass (cf. Refs. [39-41] for the corresponding expression in the 3D Georgi-Glashow model):

$$
m_{D}^{2}=\frac{\mu^{4}}{M^{2}}\left(1+\frac{\mu^{4}}{M^{4}}\right) \simeq m^{2}\left(1+\frac{m^{2}}{M^{2}}\right)
$$

Thus, with a deviation from the London limit, the DAHM vacuum correlation length $1 / m$, equal to $1 / m_{D}$, acquires a negative correction $-\frac{m^{2}}{2 M^{2}}$. Note that this decrease of the vacuum correlation length is in line with the attractive force, which is known to start acting onto two parallel same-oriented Abrikosov-Nielsen-Olesen strings away from the London limit due to the Higgs-boson interactions (in addition to the repulsive force, which is produced by the gauge-boson interactions) [42]. Such a competition between the forces which the gauge-boson and the Higgs-boson interactions excert onto two interacting topological defects is similar for Abrikosov-Nielsen-Olesen strings and for monopoles (cf. above).

Note also that the obtained decrease of the vacuum correlation length in the vicinity of the London limit parallels its strong decrease in the Bogomolny limit, where the gaugeand the Higgs-boson masses are equal to the same quantity $m_{\text {Bog }}$ [43]. Indeed, the string tension in the Bogomolny limit differs from the one in the London limit, Equation (3), by the absence of the factor $\ln \frac{M}{m}$ [25], i.e., it reads:

$$
\sigma=\frac{m_{\mathrm{Bog}}^{2}}{4 \pi} .
$$

Since, within the dual superconductor model, both Equations (3) and (11) should have the same aforementioned phenomenological value $\sigma=(440 \mathrm{MeV})^{2}$, we obtain:

$$
\frac{m_{\mathrm{Bog}}}{m}=\sqrt{\ln \frac{M}{m}} \gg 1 .
$$

Accordingly, the vacuum correlation length in the Bogomolny limit, $1 / m_{\mathrm{Bog}}$, is smaller than the vacuum correlation length in the London limit, $1 / m$, by the same large factor of $\sqrt{\ln \frac{M}{m}}$.

Let us now dualize theory (10), which will yield a potential of monopole currents away from the London limit. To this end, we write $\mathrm{e}^{-\frac{1}{4} \int_{x} F_{\mu \nu}^{2}}=\int \mathcal{D} j_{\mu} \mathrm{e}^{-\frac{1}{2} \int_{x, y} j_{\mu}(x) D_{0}(x-y) j_{\mu}(y)-i \int_{x} B_{\mu} j_{\mu}}$, where the first term in the exponential on the r.h.s. represents Coulomb interaction of monopole currents, while the dual field $B_{\mu}$ has no kinetic term anymore, and can thus be 
integrated over in the saddle-point approximation. For a rescaled field $b_{\mu} \equiv i B_{\mu} / M$, the action has the form

$$
S\left[b_{\mu}\right]=M \int_{x} b_{\mu} j_{\mu}-\mu^{4} \int_{x} \cosh \left(\left|b_{\mu}\right|\right)-\frac{\mu^{8}}{2 M^{2}} \int_{x, y} \cosh \left(\left|b_{\mu}(x)\right|\right) \cosh \left(\left|b_{\mu}(y)\right|\right) D_{M}(x-y) .
$$

Assuming $b_{\mu}$ of the form: $b_{\mu}=\frac{j_{\mu}}{\left|j_{\mu}\right|}\left|b_{\mu}\right|$, we obtain the following saddle-point equation:

$$
\sinh \left(\left|b_{\mu}(x)\right|\right)\left[1+\frac{\mu^{4}}{M^{2}} \int_{y} \cosh \left(\left|b_{\mu}(y)\right|\right) D_{M}(x-y)\right]=\frac{M\left|j_{\mu}\right|}{\mu^{4}}
$$

Seeking its solution in the form $\left|b_{\mu}\right|=\phi+\chi$, where $|\chi| \ll \phi$, we obtain in the leading approximation: $\phi_{\text {s.p. }}=\operatorname{arcsinh}\left(M\left|j_{\mu}\right| / \mu^{4}\right), \chi_{\text {s.p. }}=0$. This solution to the saddle-point equation yields the following potential of monopole currents:

$$
V\left[j_{\mu}\right]=M \int_{x}\left|j_{\mu}\right| \operatorname{arcsinh}\left(M\left|j_{\mu}\right| / \mu^{4}\right)-\mu^{4} \int_{x} f
$$

where $f(x) \equiv \sqrt{1+\left(M j_{\mu}(x) / \mu^{4}\right)^{2}}$. Retaining further the linear-in- $\chi$ terms in the saddle-point equation, we obtain its following solution: $\chi_{\text {s.p. }}(x)=-\left|j_{\mu}(x)\right| /(M f(x)) \int_{y} D_{M}(x-y) f(y)$. We explicitly see that the inequality $\left|\chi_{\text {s.p. }}\right| \ll \phi_{\text {s.p. }}$ holds at low monopole densities, $\left|j_{\mu}\right| \ll \mu^{4} / M$, in which case $\left|\chi_{\text {s.p. }}\right| / \phi_{\text {s.p. }} \simeq(\mu / M)^{4}$. Accordingly, in the corresponding expression for the potential,

$$
V\left[j_{\mu}\right]=M \int_{x}\left|j_{\mu}\right| \operatorname{arcsinh}\left(M\left|j_{\mu}\right| / \mu^{4}\right)-\mu^{4} \int_{x} f-\frac{\mu^{8}}{2 M^{2}} \int_{x, y} f(x) f(y) D_{M}(x-y),
$$

only the quadratic term should be retained in this low-density approximation. That yields

$$
V\left[j_{\mu}\right] \simeq \frac{1}{2 m_{D}^{2}} \int_{x} j_{\mu}^{2}
$$

Here, $\frac{1}{m_{D}^{2}} \simeq \frac{M^{2}}{\mu^{4}}-\frac{1}{M^{2}}$ consistently substitutes $\frac{1}{m_{D}^{2}}=\frac{M^{2}}{\mu^{4}}$ which enters the same expression for $V\left[j_{\mu}\right]$ in the case where the correction is not taken into account, i.e., $\chi_{\text {s.p. }}=0$.

\section{Summary}

This paper has been devoted to the two aspects of the dual superconductor scenario of confinement. The first one was the derivation of the string representation of a Wilson loop in the 4D Yang-Mills theory compactified on a torus. For the Gaussian approximation to the Yang-Mills theory, we have used the explicit form of the two-point correlation function of gluonic field strengths, which can be obtained in the London limit of the dual superconductor. With a deviation from the Gaussian approximation, the quartic cumulant of gluonic field strengths starts contributing to the Wilson loop. That cumulant leads to the appearance, in the string representation, of a term corresponding to the two-point correlation of the Euler characteristic of the string world sheet. At short distances, this correlation gets reduced to a massless 2D propagator, yielding the Polchinski-Strominger term (6) in the string representation. When relation (7) between the circumferences of the compactification circles holds, that term cancels string conformal anomaly in $4 \mathrm{D}$, thereby making string representation fully quantum. While we find this result interesting, we notice that its derivation relies on the mentioned explicit form of the two-point correlation function of gluonic field strengths which corresponds to the London limit of the dual superconductor. Indeed, only that correlation function yields, upon the compactification of the theory on the torus, the correlation function (2), whose short-distance logarithmic asymptotics eventually yields the Polchinski-Strominger term. We notice here one more remarkable fact stemming from the logarithmic short-distance asymptotics of the Macdonald function $K_{0}(x)$ which 
describes also the electric field of a straight-line dual Abrikosov-Nielsen-Olesen string, i.e., a flux tube, in the London limit of the dual superconductor. Namely, pairs produced in the field of such a flux tube have an exponential distribution, in contrast to the standard Schwinger's Gaussian distribution, which takes place away from the London limit $[44,45]$.

The second aspect of the dual superconductor scenario, addressed in this paper, was the deviation from the London limit. We have used the correspondence between the dual formulations of DAHM in the London limit and of the low-energy limit of $4 \mathrm{D}$ compact QED, to write down partition function (9) of DAHM away from the London limit. It is similar to the partition function of the grand canonical ensemble of monopoles in the 3D Georgi-Glashow model where the Higgs field is considered not infinitely heavy. Having integrated, in the Gaussian approximation, over the scalar field which represents in this formulation the dual Higgs field, we have obtained a decrease of the vacuum correlation length of DAHM away from the London limit, by a factor of $\left(1-\frac{m^{2}}{2 M^{2}}\right)$, where $m$ and $M$ are the masses of the dual vector boson and the dual Higgs field, respectively. Such a decrease parallels the known fact about an attractive force caused by the Higgs-boson exchanges between two parallel same-oriented straight-line dual Abrikosov-Nielsen-Olesen strings, as well as the strong decrease of the vacuum correlation length in the Bogomolny limit. Finally, by dualizing partition function (9), we have obtained its representation in terms of monopole currents, which includes Coulomb interaction of those currents along with their corresponding potential.

Funding: This research received no external funding.

Conflicts of Interest: The author declares no conflict of interest.

\section{References}

1. Mandelstam, S. Vortices and quark confinement in non-Abelian gauge theories. Phys. Lett. B 1975, 53, 476-478. [CrossRef]

2. Hooft, G. Topology of the gauge condition and new confinement phases in non-Abelian gauge theories. Nucl. Phys. B 1981, 190, 455-478. [CrossRef]

3. Ripka, G. Dual superconductor models of color confinement; Springer Verlag: Berlin/Heidelberg, Germany, 2004.

4. Di Giacomo, A.; Lucini, B.; Montesi, L.; Paffuti, G. Color confinement and dual superconductivity of the vacuum. 1. Phys. Rev. D 2000, 61, 034503. [CrossRef]

5. Di Giacomo, A.; Lucini, B.; Montesi, L.; Paffuti, G. Color confinement and dual superconductivity of the vacuum. 2. Phys. Rev. D 2000, 61, 034504. [CrossRef]

6. Antonov, D. Monopole-based scenarios of confinement and deconfinement in 3D and 4D. Universe 2017, 3, 50. [CrossRef]

7. Antonov, D. Gluodynamics string as a low-energy limit of the Universal Confining String theory. Phys. Lett. B 1998, 427, $274-282$. [CrossRef]

8. Dosch, H.G. Gluon condensate and effective linear potential. Phys. Lett. B 1987, 190, 177-181. [CrossRef]

9. Marquard, U.; Dosch, H.G. Potential and sum-rule approach in QCD. Phys. Rev. D 1987, 35, 2238. [CrossRef]

10. Di Giacomo, A.; Dosch, H.G.; Shevchenko, V.I.; Simonov, Yu.A. Field correlators in QCD: Theory and applications. Phys. Rept. 2002, 372, 319-368. [CrossRef]

11. Bicudo, P.; Brambilla, N.; Ribeiro, E.; Vairo, A. Confinement and chiral-symmetry breaking in heavy-light quark systems. Phys. Lett. B 1998, 442, 349-358. [CrossRef]

12. Polchinski, J.; Strominger, A. Effective string theory. Phys. Rev. Lett. 1991, 67, 1681-1684. [CrossRef] [PubMed]

13. Polyakov, A.M. Gauge Fields and Strings; Harwood Academic Publishers: Chur, Switzerland, 1987.

14. Akhmedov, E.T.; Chernodub, M.N.; Polikarpov, M.I.; Zubkov, M.A. Quantum theory of strings in Abelian Higgs model. Phys. Rev. D 1996, 53, 2087-2095. [CrossRef] [PubMed]

15. Antonov, D. Fully quantum string representation of a Wilson loop in the finite-temperature 3D Yang-Mills theory. Symmetry 2020, 12, 688. [CrossRef]

16. Ünsal, M.; Yaffe, L.G. Center-stabilized Yang-Mills theory: Confinement and large- $N$ volume independence. Phys. Rev. D 2008, 78, 065035. [CrossRef]

17. Chernodub, M.N.; Goy, V.A.; Molochkov, A.V. Phase structure of lattice Yang-Mills theory on $\mathbb{T}^{2} \times \mathbb{R}^{2}$. Phys. Rev. D 2019, 99, 074021. [CrossRef]

18. Wipf, A. Statistical approach to quantum field theory; Springer: Berlin/Heidelberg, Germany, 2013; Chapter 14.

19. Athenodorou, A.; Cardinali, M.; D’Elia, M. Spectrum of trace-deformed Yang-Mills theories. Phys. Rev. D 2021, 104, 074510. [CrossRef]

20. Baker, M.; Brambilla, N.; Dosch, H.G.; Vairo, A. Field-strength correlators and dual effective dynamics in QCD. Phys. Rev. D 1998, 58, 034010. [CrossRef] 
21. Antonov, D. Field correlators in Abelian-projected theories and stochastic vacuum model. JHEP 2000, 7, 55. [CrossRef]

22. Antonov, D. String nature of confinement in (non-)Abelian gauge theories. Surv. High Energy Phys. 2000, 14, 265-355. [CrossRef]

23. Mumford, D. Tata lectures on Theta I; Birkhäuser: Boston, USA, 1983.

24. Lifshitz, E.M.; Pitaevskii, L.P. Statistical physics, Part 2; Butterworth-Heinemann: Oxford, UK, 1980.

25. Antonov, D. Nonperturbative methods in gauge theories; Pisa University Press: Pisa, Italy, 2013.

26. Meggiolaro, E. Field-strength correlators in QCD: New fits to the lattice data. Phys. Lett. B 1999, 451, 414-421. [CrossRef]

27. Kornelis, W.; Dosch, H.G. Higher cumulants in the cluster expansion in QCD. Nucl. Phys. Proc. Suppl. 2001, 96, 426-431. [CrossRef]

28. Di Giacomo, A.; Panagopoulos, H. Field-strength correlations in the QCD vacuum. Phys. Lett. B 1992, 285, 133-136. [CrossRef]

29. Boyd, G.; Engels, J.; Karsch, F.; Laermann, E.; Legeland, C. Thermodynamics of SU(3) lattice gauge theory. Nucl. Phys. B 1996, 469, 419-444. [CrossRef]

30. Agasian, N.O. Thermal gluomagnetic vacuum of SU(N) gauge theory. Phys. Lett. B 2003, 562, 257-264. [CrossRef]

31. Selikhov, A.; Gyulassy, M. Color diffusion and conductivity in a quark-gluon plasma. Phys. Lett. B 1993, 316, 373-380. [CrossRef]

32. Bödeker, D. Effective dynamics of soft non-Abelian gauge fields at finite temperature. Phys. Lett. B 1998, 426, 351-360. [CrossRef]

33. Antonov, D. Exploring quark-gluon plasma on the loop space. Phys. Lett. B 2002, 545, 97-104. [CrossRef]

34. Antonov, D. Contributions of stochastic background fields to the shear and bulk viscosities of the gluon plasma. Ann. Phys. 2010, 325, 1304-1315. [CrossRef]

35. Antonov, D. Shear viscosity of a nonperturbative gluon plasma. arXiv 2012, arXiv:1202.2313.

36. Arnold, P.B.; Moore, G.D.; Yaffe, L.G. Transport coefficients in high-temperature gauge theories: (II) Beyond leading log. JHEP 2003, 5, 51. [CrossRef]

37. Polyakov, A.M. Quark confinement and topology of gauge theories. Nucl. Phys. B 1977, 120, 429-458. [CrossRef]

38. Dietz, K.; Filk, Th. Critical Higgs mass for the (2+1)-dimensional Georgi-Glashow model. Nucl. Phys. B 1980, 164, 536-545. [CrossRef]

39. Agasian, N.O.; Antonov, D. Confining strings and RG flow in the (2+1)-dimensional Georgi-Glashow model and its SU(3)generalization. JHEP 2001, 6, 58. [CrossRef]

40. Antonov, D. Accounting for the finiteness of the Higgs-boson mass in the 3D Georgi-Glashow model. Mod. Phys. Lett. A 2002, 17, 279-288. [CrossRef]

41. Antonov, D.; Diamantini, M.C. 3D Georgi-Glashow model and confining strings at zero and finite temperatures. In From Fields to Strings: Circumnavigating Theoretical Physics; World Scientific: Singapore, 2005; pp. 188-265.

42. Vilenkin, A.; Shellard, E.P.S. Cosmic Strings and Other Topological Defects; Cambridge University Press: Cambridge, UK, 1994; Chapter 4.3.

43. Bogomolny, E.B. Stability of classical solutions. Sov. J. Nucl. Phys. 1976, 24, 449-454.

44. Antonov, D.; Di Giacomo, A. String breaking in QCD: dual superconductor vs. stochastic vacuum model. JHEP 2005, 3, 17. [CrossRef]

45. Antonov, D. Calculating non-perturbative quantities through the world-line formalism. J. Phys. Conf. Ser. 2019, 1208, 012005. [CrossRef] 